\title{
Evidence against visual integration across saccadic eye movements
}

\author{
DAVID E. IRWIN \\ Cornell University, Ithaca, New York \\ and \\ STEVEN YANTIS and JOHN JONIDES \\ University of Michigan, Ann Arbor, Michigan
}

\begin{abstract}
One of the classic problems in perception concerns how we perceive a stable, continuous visual world even though we view it via a temporally discontinuous series of eye movements. Previous investigators have suggested that our perception of a stable visual environment is due to an integrative visual buffer, a special memory store capable of fusing the visual contents of successive fixations according to their environmental coordinates. In this paper, three experiments are reported that attempted to demonstrate the existence of an integrative visual buffer. The experimental procedure required subjects to mentally fuse two halves of a dot matrix presented in the same spatial region of a display, but separated by an eye movement so that each half was viewed only during one fixation. Thus, subjects had to integrate packets of visual information that had the same environmental coordinates, but different retinal coordinates. No evidence was found in any experiment for the fusion of visual information from successive fixations in memory, leaving the status of the integrative visual buffer in serious doubt.
\end{abstract}

The visual world contains more information than we can perceive in a single glance. To compensate for this problem, our eyes scan the world by means of saccades-fast, ballistic movements that are interspersed with brief fixations during which the eyes are relatively still. But this mixture of saccades and fixations introduces several problems that the human perceptual system must solve in order to operate as it does. One problem arises from differences in the quality of visual information that is available during saccades and fixations. During a saccade, visual stimulation sweeps across the retina at velocities of at least 500-600 deg/sec (Robinson, 1964), producing a blur or smear that we do not ordinarily perceive because of saccadic suppression (Campbell \& Wurtz, 1978). Thus, in some sense, our visual information about the world is registered in isolated glimpses separated in time. A further complication is that the

This paper is based on portions of a dissertation submitted by D. E. Irwin to the University of Michigan in partial fulfillment of the requirements for the doctoral degree. The research was funded by a grant from the Air Force Office of Scientific Research. D. E. Irwin was supported by a Rackham predoctoral fellowship from the University of Michigan, and S. Yantis was supported by a National Science Foundation graduate fellowship. We would like to thank John Palmer and James Zacks for helpful advice, Richard Abrams, Carol Pluzinski, and John Sullivan for technical assistance, and Vincent Di Lollo, Keith Rayner, and an anonymous reviewer for their comments on an earlier version of the manuscript. Requests for reprints may be directed to David E. Irwin, Department of Psychology, Uris Hall, Cornell University, Ithaca, New York 14853. retinal positions of objects in the world change suddenly and drastically when the eyes move. Yet, despite these difficulties, we ordinarily see the world as unified, stable, and continuous. For years, psychologists have wondered about the processes that permit us to perceive the world as a coherent whole despite such temporally discontinuous visual input.

Helmholtz (1866) was among the first to note that objects in space appear to maintain a constant location with respect to the viewer even though their positions on the retina change when the eyes move. This phenomenon, usually called visual direction constancy, suggests that we are somehow able to attend to or compute the environmental or spatial (as opposed to retinotopic) coordinates of objects in the world. Some investigators have even suggested that visual information from a previous fixation might be carried across an eye movement and combined with the contents of a new fixation, according to spatial, rather than retinal, coordinates. McConkie and Rayner (1976) have made this argument most clearly; they suggested that while a new fixation overrides the retinal activity pattern of a prior fixation, at a higher level in the perceptual system the contents of the two fixations may be spatially integrated into a single representation of the stimulus. This integration takes place in what they have called the integrative visual buffer (IVB). This structure differs from "iconic memory" (Neisser, 1967) in that more than one fixation can be stored, and information is coded spatially rather than retinotopically. 
The concept of an integrative visual buffer is intuitively appealing; a structure or a set of processes capable of fusing visual information from successive fixations according to environmental coordinates would explain in a satisfying way our perception of a clear, stable, continuous world despite changes in eye position. But empirical evidence regarding the IVB is scanty at present. McConkie, Rayner, and their colleagues have found little evidence to support the existence of an IVB in reading (e.g., McConkie \& Zola, 1979; Rayner, McConkie, \& Zola, 1980). However, one might argue that an IVB is optional or even unnecessary in reading, since highly overlearned verbal codes and word meanings would presumably be more important than visual information per se in this situation.

In fact, other studies that have employed purely visual stimuli are more suggestive. For example, Experiment 2 of Davidson, Fox, and Dick (1973) investigated whether two stimuli presented at the same spatial location, but separated by a saccade, would appear to be simultaneously available to a viewer. On each trial, a circle and a square were presented for $60 \mathrm{msec}$ just before the occurrence of a saccade; 50 $120 \mathrm{msec}$ later, a vertical and a horizontal line segment were presented for $60 \mathrm{msec}$ in the same spatial area. Subjects had to report whether the line segments spatially overlapped the circle and the square or were next to them. Davidson et al. found that subjects were almost perfect in making this judgment. Furthermore, they reported that most of the stimuli seemed to "overlap" in time if the interstimulus interval was less than $85 \mathrm{msec}$ or so, although they never appeared to be simultaneously present.

Ritter (1976) employed a similar procedure with even simpler stimuli-brief flashes of light. A light was flashed for $10 \mathrm{msec} 4 \mathrm{deg}$ from fixation just before the onset of a saccade, and then flashed again sometime after the saccade ended. The two light flashes were presented at the same spatial location, but since they were separated by an eye movement, they stimulated different retinal locations. Ritter found that if the interval between the two flashes was less than about $75 \mathrm{msec}$, the two flashes were perceived as one. At longer intervals, two separate flashes were seen. This study, along with that of Davidson et al. described above, suggests the existence of a briefly lasting memory in which visual information from successive fixations is stored according to its spatial, and not its retinal, coordinatesthat is, in an integrative visual buffer.

While these studies are suggestive, the fact that they are based primarily on introspective reports is somewhat troubling. Furthermore, the Davidson et al. task may have been so easy that their results could have been obtained without spatial integration, and Ritter's results can be explained in terms of saccadic suppression. There are, however, a few other studies that have employed more objective response measures. One such study was performed by Wolf, Hauske, and Lupp (1978). These investigators had subjects discriminate between gratings that varied in spatial frequency following a saccade to a target location. They found that the threshold for medium spatial frequency targets decreased if an identical spatial frequency "priming" stimulus was presented at the same location just prior to the saccade. This finding suggests a position-specific integration of information from the periphery of one fixation and the fovea of the next. This conclusion was supported by a subsequent study (Wolf, Hauske, \& Lupp, 1980) that found that misaligning the prime and the target by 0-180 deg of phase led to a monotonic decrease in performance. Thus, these two studies by Wolf et al. provide support for the existence of a visual memory in which information from successive fixations is stored in proper spatial registry. It should be noted, however, that the phosphor used by Wolf et al. (P31 ) is known to decay relatively slowly, so it is entirely possible that their results were due to phosphor persistence, rather than to persistence in memory.

Another study that may suggest the existence of an integrative visual buffer was conducted by Jonides, Irwin, and Yantis (1982). The task employed in that experiment was adapted from one designed by Di Lollo $(1977,1980)$ to study integration within a single fixation. Subjects were required to remember a visual pattern that appeared during only one fixation, and then combine it with a visual pattern that appeared only after an eye movement. That is, 24 of 25 dots from a $5 \times 5$ dot matrix were presented in two frames of time, 12 dots per frame. The two frames were presented in the same spatial region of a display, but the subjects saw only one frame during one fixation and only the second frame in a subsequent fixation. Specifically, frame 1 was presented in the visual periphery, and frame 2 was seen at the fovea. The subject's task was to identify the location, in the $5 \times 5$ matrix, in which no dot had been presented. Thus, this task required that subjects integrate the two frames of dots according to their spatial, and not their retinal, coordinates. To assess subjects' integration performance, a control condition was included in which the retinal information about the two frames was identical to that described above, but the frames did not spatially overlap. That is, frame 1 was presented in the periphery and frame 2 at the fovea while the subject kept his eyes fixed in the center of the display.

Jonides et al. (1982) found that accuracy in the saccade condition was nearly 10 times as great as accuracy in the control condition, even though the retinal information in the two conditions was roughly identical. Thus, this experiment implies that packets of information with the same spatial coordinates, but different retinal coordinates, are stored simulta- 
neously in a spatially reconciled form. Unfortunately, recent tests of the graphics display device used by Jonides et al. have raised the possibility that the integration performance observed was due to persistence on the screen of the CRT rather than in the subjects' visual system.

An experiment reported by Breitmeyer, Kropfl, and Julesz (1982), who used a procedure similar to that of Jonides et al. (1982) in that subjects were required to integrate information from two successively presented frames of dots, also lends support to the integration hypothesis. The Breitmeyer et al. study has a serious drawback, however: eye movements were not monitored in the course of the experiment. The duration of the first frame was fixed at $200 \mathrm{msec}$, and that of the interstimulus interval at $40 \mathrm{msec}$. It is entirely possible, indeed likely, that a subject could initiate and complete a saccade in less than $200 \mathrm{msec}$, especially with practice. We found, for instance, that although subjects usually had a saccade latency of about 200 msec during their first experimental session, they quickly became faster and stabilized at a lower level. Furthermore, there is considerable variability about the mean latency. Thus, Breitmeyer et al. have no way of determining, on any given trial, whether or not subjects saw both frames of dots in the same fixation. It could be that trials on which subjects made a correct response were trials on which the subjects had made a fast saccade and hence seen both frames of dots in the same fixation. This likelihood considerably weakens the conclusions Breitmeyer et al. wish to draw concerning the nature of integration.

Given the ambiguity of the results of Jonides et al. (1982) and of others, we felt it necessary to attempt a replication of our earlier experiment under conditions that could unambiguously test the integration hypothesis, in particular, under conditions in which screen persistence could not contribute to any integration effect. The current paper is a complete report of experiments described briefly elsewhere (Jonides, Irwin, \& Yantis, in press).

\section{EXPERIMENT 1}

\section{Method}

Subjects. Five subjects participated in this experiment. Subject 1 was aware of the hypotheses under study, but was inexperienced as a subject in eye-movement experiments. Subject 2 had had previous experience as a subject in eye-movement studies, and he was aware of the purpose of the experiment. Subjects 3, 4, and 5 had had no prior experience in eye-movement experiments, and were completely naive about the hypotheses and predictions of this experiment.

Apparatus. A display consisting of 53 amber light-emitting diodes (LEDs) was constructed for this experiment. The LEDs were mounted between thin sheets of pegboard, and opaque foam rubber was placed around the individual LEDs so that their illuminance was restricted to a specific spatial location. A thin metal sheet containing tiny holes that corresponded to each LED loca- tion was placed over the pegboard. The illuminance of a frame of 12 dots displayed with this device was $4.1 \mathrm{~lx},{ }^{\prime}$ and the background luminance in the room was $0.5 \mathrm{~cd} / \mathrm{m}^{2}$. The center of the matrix was $4.2 \mathrm{deg}$ from fixation, and the entire matrix was $2.8 \mathrm{deg}$ in width (so the nearest edge was 2.7 deg from fixation).

The LEDs were controlled by a Digital Equipment Corporation PDP-11/60 computer via a digital input/output interface. Fifty of the LEDs were arranged as two adjacent, nonoverlapping $5 \times 5$ matrices; in addition, for calibration purposes, one LED was placed to the left of the leftmost matrix, one between the two matrices, and one to the right of the rightmost matrix. All dots were $.7 \mathrm{deg}$ apart horizontally and vertically.

The computer was also used to record responses typed into the keyboard of a Hewlett-Packard 2621A terminal. Furthermore, it recorded the output from a Gulf and Western Model 200 scleral reflectance eye monitor via an analog-to-digital converter. The eye monitor was configured to record from the right eye only, and was calibrated to be sensitive to only horizontal movements of the eye. The eye monitor was mounted on eyeglass frames that were held snugly in place on subjects' heads via a headband. During the experiment, subjects were seated $43 \mathrm{~cm}$ from the graphics scope and used a bitebar with dental impression compound to keep their heads steady.

Procedure. There were two conditions in this experiment. Figure 1 illustrates the sequence of events in each condition. In the main condition of interest, the saccade condition, the events on each trial were as follows: First, a fixation dot appeared in the center of the leftmost dot matrix. When ready, the subject pressed a key on the keyboard in front of him or her which initiated the trial. Following the keypress, a variable interval of time elapsed (mean $=1,400 \mathrm{msec}$, range $=600-2,200 \mathrm{msec}$ ), after which the fixation dot disappeared and the display was blank for $100 \mathrm{msec}$. After this, the first frame of 12 dots, chosen randomly from the rightmost $5 \times 5$ dot matrix, appeared to the right of fixation. The appearance of frame 1 was the cue to the subject to execute a saccade to the matrix location. Accordingly, the duration of the first frame was individually chosen for each subject to approximate his or her mean latency to initiate a saccade to the matrix location (these values were $180,150,200,200$, and $190 \mathrm{msec}$ for Subjects 1-5, respectively). Thus, on the average, frame 1 disappeared from view just as the subject initiated the saccade to the new location.

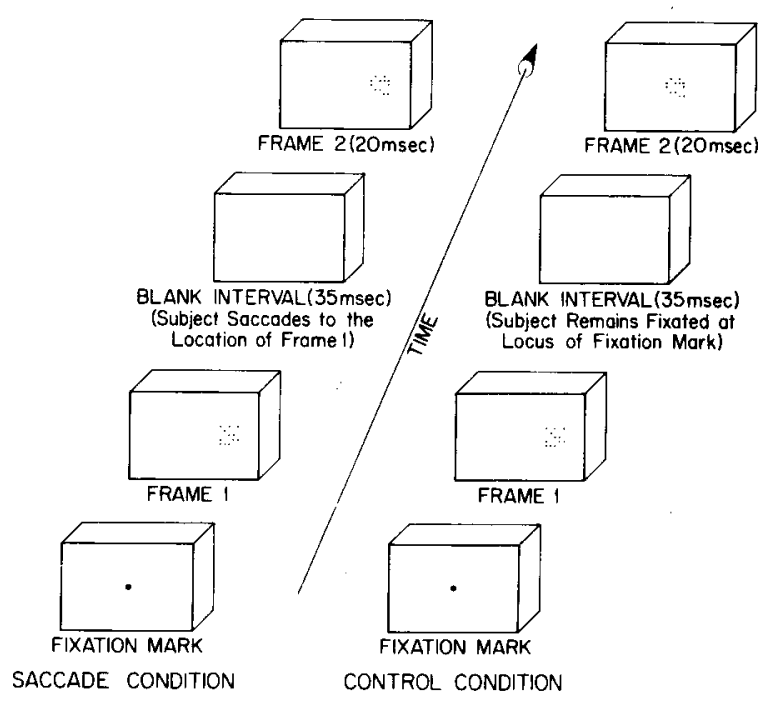

Figure 1. Schematic illustration of the experimental procedure. If the two frames in this illustration are superimposed, the missing dot location is in the second column, third row. 
Following frame 1 offset, the screen was blank for $35 \mathrm{msec}$, approximately the average duration of subjects' saccades in this experiment. Then the second frame of 12 dots appeared for $20 \mathrm{msec}$ in the same location as the first frame, such that if the two frames were physically superimposed they would form 24 of 25 dots from a $5 \times 5$ dot matrix. Following frame 2 presentation, the subject indicated the location of the missing dot by entering its row and column coordinates into a keyboard. Feedback about response accuracy and the location of the missing dot was given after each trial, and then the fixation dot reappeared for the next trial.

The second condition in this experiment was a control condition that mimicked the retinal display of the saccade condition but required no eye movements. In the control condition, the first frame appeared at the same position as in the saccade condition, but the second frame appeared where the fixation mark had been. The subject was instructed not to move his or her eyes in this condition; therefore, frame 1 appeared in the right periphery, and frame 2 appeared at the fovea, just as in the saccade condition. The time parameters of the display for this condition were yoked to those of the saccade condition for each subject. The critical difference between the two conditions was that the frames spatially overlapped in the saccade condition but not in the control condition, so that if subjects were able to spatially integrate the two frames their performance should be considerably better in the saccade condition. The control condition yields an estimate of integration accuracy based on retinal information alone; it also provides an estimate of chance guessing that is more appropriate than the pure guessing estimate of $1 / 25$, since subjects may remember some few of the actual dot positions from either frame separately and thereby increase their chances of guessing from among the rest of the locations, even though they might not have integrated the two frames at all.

After several blocks of practice trials with both conditions, the subject completed several sessions of the saccade condition and at least one control session. The subject began each session by having the eye monitor mounted on his or her head and the sensors on the monitor adjusted so that 20-deg excursions of the eyes in either direction produced maximal output from the monitor. Following this, five blocks of 15 trials each were run in a 30 -min session. Before each block of 15 trials, the subject completed four calibration trials. Each calibration trial consisted of a dot that stepped across the display at five locations separated by $2.1 \mathrm{deg}$ from each other. The leftmost of these was 2.1 deg to the left of the location used for the fixation mark during the test trials, and the rightmost was $0.7 \mathrm{deg}$ to the right of the rightmost edge of the dot matrix. On two calibration trials, the dot stepped to the right, and on two trials it stepped to the left. During calibration trials, the subject was required to fixate the dot at each position. Eye position was sampled for $100 \mathrm{msec}$ at each location. These recordings served as the basis for calibrating the output of the eye monitor against spatial position.

Following calibration, the experimental trials began. The subject maintained his or her position on the bitebar during each block of 15 trials, but was allowed to rest between blocks. Missing dot locations were drawn equally often from the 25 available positions during each session of 75 trials. During each trial, $400 \mathrm{msec}$ of eye position sampling was taken, starting with the presentation of frame 1 . Eye position was sampled 10 times/msec, and every 10 samples were averaged to obtain a 1-msec running estimate of eye position.

Persistence test. According to the manufacturer, the fall time of the LEDs is much less than $1 \mathrm{msec}$. However, in order to ensure that the LEDs did not persist after their nominal offset, Subjects 1 and 2 also participated in the following psychophysical test. A closed tachistoscopic shutter was placed over the LED display. On a random half of the test trials, a single LED was illuminated for $100 \mathrm{msec}$ and then extinguished. Immediately following LED offset, the shutter opened. On the other test trials, the LED was not illuminated but the shutter merely opened at the appropriate point in the trial sequence. The subjects' task was to say whether an LED had been illuminated or not, which should be a trivial decision if there was LED persistence.
The psychophysical tests of LED persistence yielded the following results. Subject 2 reported never seeing any trace of LED persistence on 96 test trials. Subject 1 adopted a looser criterion that resulted in 4 hits, 28 misses, 5 false alarms, and 27 correct rejections, corresponding to a d', or sensitivity, of -0.14 . Thus, we can be fairly confident that there was no LED persistence in this experiment that might create spurious integration.

Data analysis. Timing was of critical importance in these experiments; therefore, measurements of the delay in signal transmission from the eye monitor and in illuminating and extinguishing LEDs in the display were obtained and taken into account during data analysis. The output of the eye monitor was not analyzed on-line, but, rather, was stored and analyzed after each experimental session was completed.

Since the stimuli were not presented and removed contingent on eye position, the trials of the saccade condition were of four types: those on which the subject shifted his or her gaze too quickly and therefore saw both frames in the same fixation foveally; those on which the subject shifted his or her gaze too slowly and consequently saw both frames in the same fixation peripherally; those on which the subject shifted his or her gaze too slowly so that the first frame was presented during one fixation and the second frame only during the saccade; and those on which the subject executed a saccade within a proper window of time such that he or she saw the first frame during one fixation (and, perhaps, during the saccade as well, if any visual information is processed then) and the second frame during the second fixation. These last trials will be called "window" trials, to indicate that the saccade occurred during the proper window of time. Since our interest centers chiefly on the window trials, the data of the saccade condition were partitioned into these four types of trials. Three criteria were used for identifying window trials: (1) The saccade must have ended after the offset of frame 1 ; (2) the saccade must have started before the onset of frame 2; and (3) the saccade must have ended before the offset of frame 2 .

\section{Results and Discussion}

Table 1 presents the frequencies of each of the trial types for each subject and the accuracy of missing-dot localization for each type of trial as well. Data from the control condition are included for comparison.

Type 1 and Type 2 trials are ones on which the two frames overlapped retinally and spatially; that is, both frames were viewed during the same fixation. Hence, they are not of major interest in this study, which concerns integration of visual information from two separate fixations. They do, however, serve as replications of Di Lollo's $(1977,1980)$ experiments on temporal integration within a single fixation, and yield results comparable to his. Type 3 trials are also of little interest here, since frame 2 was presented during the saccade and may not have been seen clearly. The trials that are of major interest here are those of Types 4 and 5. Table 1 shows that performance in the window condition was no better than performance in the control. That is, subjects were unable to integrate the two dot matrices when they were viewed during different fixations. Thus, the results of Jonides et al. (1982) were not replicated. In an attempt to uncover any trace of an integration effect, the overall results of this experiment were broken down and analyzed more closely.

Table 2 shows accuracy at identifying the missing dot as a function of the eyes' landing time in the 
Table 1

Accuracy (in Percent) and Frequency for Each Type of Trial in Experiment 1 by Subject

\begin{tabular}{|c|c|c|c|c|c|c|c|c|c|c|}
\hline \multirow[b]{2}{*}{ Trial Type } & \multicolumn{2}{|c|}{ Subject 1} & \multicolumn{2}{|c|}{ Subject 2} & \multicolumn{2}{|c|}{ Subject 3} & \multicolumn{2}{|c|}{ Subject 4} & \multicolumn{2}{|c|}{ Subject 5} \\
\hline & $\mathrm{N}$ & A & $\mathrm{N}$ & A & $\mathrm{N}$ & $\mathbf{A}$ & $N$ & A & $\mathbf{N}$ & A \\
\hline End Too Early & 7 & 28.6 & 25 & 48.0 & 47 & 21.3 & 15 & 0.0 & 83 & 60.2 \\
\hline Start Too Late & 14 & 21.4 & 130 & 12.3 & 65 & 16.9 & 32 & 9.4 & 17 & 17.6 \\
\hline End Too Late & 0 & 0.0 & 27 & 7.4 & 1 & 0.0 & 12 & 8.3 & 3 & 0.0 \\
\hline Window & 99 & 10.1 & 388 & 8.5 & 112 & 11.6 & 115 & 7.8 & 125 & 15.2 \\
\hline Control & 102 & 5.9 & 116 & 6.9 & 73 & 13.7 & 63 & 7.9 & 133 & 9.8 \\
\hline
\end{tabular}

Note $-N=$ frequencies: $A=$ accuracy.

Table 2

Accuracy (in Percent) and Frequency as a Function of Eye Landing Time in the Window for Each Subject in Experiment 1

\begin{tabular}{|c|c|c|c|c|c|c|c|c|c|c|}
\hline \multirow{2}{*}{$\begin{array}{l}\text { Landing } \\
\text { Time* }\end{array}$} & \multicolumn{2}{|c|}{ Subject 1} & \multicolumn{2}{|c|}{ Subject 2} & \multicolumn{2}{|c|}{ Subject 3} & \multicolumn{2}{|c|}{ Subject 4} & \multicolumn{2}{|c|}{ Subject 5} \\
\hline & $\mathbf{N}$ & A & $\mathbf{N}$ & A & $\mathrm{N}$ & A & $\mathbf{N}$ & A & $\mathrm{N}$ & A \\
\hline $1-10$ & 20 & 25.0 & 27 & 22.2 & 17 & 11.8 & 23 & 13.0 & 47 & 17.0 \\
\hline $11-20$ & 25 & 4.0 & 46 & 2.2 & 19 & 15.8 & 32 & 6.2 & 35 & 8.6 \\
\hline $21-30$ & 30 & 13.3 & 113 & 7.1 & 28 & 3.6 & 16 & 6.2 & 25 & 16.0 \\
\hline $31-40$ & 15 & 0.0 & 122 & 9.8 & 27 & 18.5 & 20 & 5.0 & 11 & 36.4 \\
\hline $41-50$ & 9 & 0.0 & 80 & 7.5 & 21 & 9.5 & 24 & 8.3 & 7 & 0.0 \\
\hline
\end{tabular}

Note $-N=$ frequencies; $A=$ accuracy. $\quad{ }^{*}$ In milliseconds.

critical window separating the two frames of dots. It may be that integration is possible at some landing times, but not at others. Three of the five subjects showed a slightly higher tendency to be correct when they landed at the earliest moment in the window. This result was somewhat unexpected, because, if information from frame 1 decays with time, one might expect integration to be poorest when the eyes land early in the window, since that information would have to be held in memory longer. Higher accuracy early in the window thus seems most likely to be due to variability in the timing measurements used in data analysis; that is, some of the early "window" trials may actually be trials in which the eyes landed before the first frame had disappeared-that is, "too early" trials. In any even, these accuracy levels are not nearly as high as those obtained by Jonides et al. (i.e., 60\%). Accuracy for other landing times in the window was no better than chance.

Subjects' poor performance on window trials in this experiment is perhaps made especially clear when the relationship between the row containing the missing dot and the subject's row response, and likewise the relationship between the column containing the missing dot and the subject's column response, are compared. Contingency tables of these relationships for window trials were constructed and then tested for independence; for rows, the $\mathrm{G}^{2}$ goodness-of-fit statistics (with $16 \mathrm{df}$ ) for each subject were 22.2, 27.7. 23.6, 37.1, and 30.6, while for columns these values were $6.8,14.6,8.8,30.4$, and 20.0 (chi-square at the .05 level of significance and $16 \mathrm{df}=26.3$ ). Thus, subjects' row and column responses were largely independent of where the target actually ap- peared. This analysis is a fairly liberal test of any integrative knowledge that subjects might have, since it does not require subjects to correctly identify the missing dot, but just come close to its row or column location. But even this liberal analysis failed to provide evidence for integration.

One final analysis of interest concerns subjects' memory for the contents of each frame of dots. When a subject made an error in this task, he or she reported a location that actually contained a dot in one of the two frames. It is worthwhile to determine which frame contained a dot at the erroneously reported location, since this is an indication of which frame is more available to the subject.

Table 3 displays errors by frame for each subject in each of the two conditions. In this table, the error data for each subject have simply been partitioned according to whether the subject reported a location that contained a dot in frame 1 or in frame 2. Thus, percentage of errors for frame 1 plus frame 2 for each subject equals $100 \%$.

Table 3

Percentage of Error Responses From Each Frame of the Matrix for Window and Control Trials in Experiment 1

\begin{tabular}{lrrrrr} 
& \multicolumn{5}{c}{ Subject } \\
\cline { 2 - 6 } & 1 & 2 & 3 & 4 & 5 \\
\hline Frame 1 & 94.4 & 90.4 & 77.2 & 79.2 & 92.5 \\
Frame 2 & 5.6 & 9.6 & 22.8 & 20.8 & 7.5 \\
& \multicolumn{5}{c}{ Control Trials } \\
Frame 1 & 99.0 & 89.8 & 76.2 & 67.2 & 81.7 \\
Frame 2 & 1.0 & 10.2 & 23.8 & 32.8 & 18.3 \\
\hline
\end{tabular}


In Jonides et al. (1982), when subjects made an error they tended, in the saccade condition, to choose a location from frame 2 more often than from frame 1. The reverse was true in the control condition. This was interpreted as evidence for frame 1's being better stored in memory when a saccade occurred. In the current experiment, almost all errors came from frame 1 and the comparison between the saccade and control conditions yielded strikingly similar results. Thus, subjects' poor performance in this task seems largely due to their failure to remember information from frame 1 (cf. Di Lollo, 1980). Rather, they adopted the strategy of guessing from among the locations that were not filled in frame 2. This guessing strategy would account for the obtained accuracy levels of slightly higher than the $4 \%$ that one would expect from guessing among 25 locations. There was no evidence at all that information from frame 1 was stored in a special memory and then integrated with the contents of frame 2 .

In summary, there was no evidence in this experiment that visual information from one fixation can be carried across an eye movement and fused, according to spatial coordinates, with the contents of a second fixation. Thus, the results of this experiment cast doubt on the existence of an integrative visual buffer, and suggest that the results of Jonides et al. (1982) were due to phosphor persistence.

There is another possible explanation for the failure to replicate Jonides et al., however. The LED display used in the present experiment was subtly different from the graphics scope used by Jonides et al. The LED display was slightly narrower than the display presented on the graphics scope, and the dots were of a different color. The graphics scope provided a smooth, continuous surface for stimulus presentation, while the LED display was more complex. In order to eliminate some of these potentially confounding visual factors, in Experiment 2 the graphics display used by Jonides et al. (1982) was again used. The only difference was that two filters were placed over the scope in order to attenuate the long persistence component of the phosphor.

\section{EXPERIMENT 2}

\footnotetext{
Method

Subjects. Subjects 1 and 2 from Experiment 1 participated in this experiment. In addition, a new Subject 3 , inexperienced in eye-movement experiments and naive to the purposes of this experiment, also participated.

Apparatus. Stimuli were presented on the face of a Digital Equipment Corporation VT-11 graphics scope equipped with a P-4 phosphor. According to the manufacturer, this phosphor decays to $1 \%$ of its initial intensity within $0.5 \mathrm{msec}$ of display offset and to $0.1 \%$ of its initial intensity within $20 \mathrm{msec}$ of display of fset.

The P-4 phosphor used in the graphics display device has two spectral components, one centered at $470 \mathrm{~nm}$ (blue) and the other at $580 \mathrm{~nm}$ (yellow). The yellow component is primarily responsible for the long decay time of the P-4. Thus, in order to attenuate the
}

visibility of the persisting phosphor, we attempted to eliminate the yellow component by placing two filters (Wratten $49 \mathrm{~A}$ and 49B) over the face of the graphics scope. The stimulus dots appeared blue when viewed through these filters. The mean illuminance of a frame of 12 dots displayed under these conditions was $3.8 \mathrm{~lx}$, and the background luminance was as before $\left(0.5 \mathrm{~cd} / \mathrm{m}^{2}\right)$.

The center of the dot matrix was $4.0 \mathrm{deg}$ from fixation, and the entire matrix was 3.0 deg in width.

Procedure. The procedure followed that of Experiment 1. The subjects completed several sessions of the saccade condition and at least one control session.

Persistence test. In order to ensure that the filters eliminated any phosphor persistence, the following test was performed. Twelve dots were presented behind a closed tachistoscopic shutter for $150 \mathrm{msec}$, then extinguished. Shortly after their offset, the shutter opened and 12 more dots were presented. Subjects tried to identify which dot had not been presented.

Subjects 2 and 3 both participated in the shutter test. Subject 2 was correct on 5 of $50(10 \%)$ trials when the shutter opened 1 msec after frame 1 offset, 5 of $50(10 \%) 8 \mathrm{msec}$ after frame 1 offset, and 4 of $50(8 \%) 16 \mathrm{msec}$ after frame 1 offset. Subject 3 was 1 of 50 $(2 \%), 6$ of $50(12 \%)$, and 3 of $50(6 \%)$ at these same three delays. Thus, the filters seemed to effectively eliminate most phosphor persistence.

\section{Results and Discussion}

Table 4 shows the results of this experiment. As in Experiment 1, performance on window trials was no better than performance in the control condition. Thus, using virtually the same display that produced integration in Jonides et al. (1982), no evidence was found for integration in this experiment. The only difference between the two displays was that filters that eliminated phosphor persistence were placed over the graphics scope in this experiment (although this did change the color of the dots as well).

Closer examination of the data verifies the lack of integration suggested by Table 4. Table 5 shows accuracy as a function of when the eyes landed in the window; performance was poor throughout the entire interval. Subjects' poor performance on window trials was further demonstrated by contingency table analyses showing that subjects' row and column responses were largely independent of the targets' actual row and column locations: For rows, tests of independence (with $16 \mathrm{df}$ ) yielded $\mathrm{G}^{2} \mathrm{~s}$ of 29.6, 13.4, and 21.2 for the three subjects, while for columns $G^{2}=22.4,17.0$, and 23.5 (chi-square at the .05 level of significance and $16 \mathrm{df}=26.3$ ).

Table 4

Accuracy (in Percent) and Frequencies for Each Type of Trial in Experiment 2 by Subject

\begin{tabular}{lrrrrrrrr}
\hline & \multicolumn{2}{c}{ Subject 1} & & \multicolumn{2}{c}{ Subject 2 } & & \multicolumn{2}{c}{ Subject 3 } \\
\cline { 2 - 3 } Trial Type & N & A & & N & A & & N & A \\
\hline End Too Early & 97 & 41.2 & & 29 & 58.6 & & 105 & 21.9 \\
Start Too Late & 14 & 0.0 & & 75 & 25.3 & 65 & 6.2 \\
End Too Late & 17 & 11.8 & & 35 & 8.6 & 23 & 0.0 \\
Window & 216 & 12.0 & & 161 & 8.7 & 169 & 10.7 \\
Control & 157 & 10.2 & & 75 & 9.3 & 105 & 7.6 \\
\hline
\end{tabular}

Note $-N=$ frequencies; $A=$ accuracy. 
Table 5

Accuracy (in Percent) and Frequencies as a Function of Eye Landing Time in the Window for Each Subject in Experiment 2

\begin{tabular}{|c|c|c|c|c|c|c|}
\hline \multirow{2}{*}{$\begin{array}{l}\text { Landing } \\
\text { Time* }\end{array}$} & \multicolumn{2}{|c|}{ Subject 1} & \multicolumn{2}{|c|}{ Subject 2} & \multicolumn{2}{|c|}{ Subject 3} \\
\hline & $\mathbf{N}$ & $\mathbf{A}$ & $\mathrm{N}$ & A & $\mathrm{N}$ & $\mathbf{A}$ \\
\hline $1-10$ & 38 & 10.5 & 11 & 0.0 & 61 & 16.4 \\
\hline $11-20$ & 41 & 12.2 & 35 & 5.7 & 35 & 14.3 \\
\hline $21-30$ & 51 & 15.7 & 46 & 8.7 & 26 & 7.7 \\
\hline $31-40$ & 48 & 14.6 & 33 & 6.1 & 24 & 4.2 \\
\hline $41-50$ & 38 & 5.3 & 36 & 16.7 & 23 & 0.0 \\
\hline
\end{tabular}

Note $-N=$ frequencies; $A=$ accuracy. $\quad$ *In milliseconds.

Table 6

Percentage of Error Responses From Each Frame of the Matrix for Window and Control Trials in Experiment 2

\begin{tabular}{cccc}
\hline & \multicolumn{3}{c}{ Subject } \\
\cline { 2 - 4 } & \multicolumn{4}{c}{2} & 3 \\
\hline \multicolumn{4}{c}{ Window Trials } \\
Frame 1 & 90.5 & 91.8 & 80.8 \\
Frame 2 & 9.5 & 8.2 & 19.2 \\
& Control Trials & \\
Frame 1 & 91.5 & 88.2 & 78.4 \\
Frame 2 & 8.5 & 11.8 & 21.6 \\
\hline
\end{tabular}

Further evidence that performance on window trials was little different from that in the control condition is illustrated in Table 6, which presents frame errors for the two conditions. The vast majority of errors in both conditions were due to subjects' reporting as missing a dot that was actually presented in frame 1 . It was as if subjects had very poor knowledge of the contents of frame 1 , so that they just ruled out the contents of frame 2 and guessed fairly randomly from the remaining 13 locations on each trial in both conditions. So there was no indication at all that information from frame 1 was specially stored when a saccade was executed and then subsequently integrated with the contents of frame 2 . Rather, information from frame 1 appeared to be lost when a saccade occurred.

Experiments 1 and 2 considered together suggest that the integration found by Jonides et al. (1982) was due to phosphor persistence on their graphics scope. In the absence of any phosphor persistence, there is no indication that visual information is integrated from successive fixations. It is possible, however, that the task subjects had to perform in Experiments 1 and 2 was just too difficult to reveal whether integration had, in fact, occurred; perhaps, with a simpler task, subjects may be capable of integrating information from two frames viewed during different fixations. The task used in Experiments 1 and 2 required subjects to reconcile two sets of 12 dots each very accurately; since the distance between adjacent dots was only $0.7-0.75 \mathrm{deg}$, even a slight error in spatial reconciliation might have made subjects' identification response impossibly difficult. Thus, in Experiment 3 an easier version of the dot integration task was employed. Here subjects tried to identify which one of nine dots in a $3 \times 3$ dot matrix was not presented. This task should be easier because there would be less information to remember and the individual dots would be farther apart, leaving more room for reconciliation error.

\section{EXPERIMENT 3}

\section{Method}

Subjects. Subjects 1 and 3 from Experiment 1 participated in this study.

Apparatus. The LED display that was used in Experiment 1 was used in this experiment. The target matrix consisted of three rows and three columns. The top, middle, and bottom rows, and the left, middle, and outermost columns of the LED display were used. Horizontal and vertical interdot distances were 1.4 deg.

Procedure. The procedure followed that of Experiment 1, except that the target matrix was presented in two frames of four dots each. Each subject participated in saccade and control conditions.

\section{Results and Discussion}

Table 7 contains the results of this experiment. Accuracy on both window and control trials was substantially higher than in previous experiments, but window trial performance was once again no better than control condition performance. As in the previous experiments, there was little effect on accuracy of the time when the eyes landed in the window (Table 8). Most errors came from frame 1 rather than frame 2 in both conditions (Table 9), once again

\section{Table 7}

Accuracy (in Percent) and Frequencies for Each Type of Trial in Experiment 3 by Subject

\begin{tabular}{lrrrrr}
\hline & \multicolumn{2}{c}{ Subject 1} & & \multicolumn{2}{c}{ Subject 2} \\
\cline { 2 - 3 } \cline { 6 - 6 } Trial Type & $\mathrm{N}$ & $\mathrm{A}$ & & $\mathrm{N}$ & $\mathrm{A}$ \\
\hline End Too Early & 31 & 71.0 & & 73 & 61.6 \\
Start Too Late & 18 & 44.4 & & 11 & 36.4 \\
End Too Late & 1 & 0.0 & & 0 & 0.0 \\
Window & 100 & 41.0 & 66 & 47.0 \\
Control & 73 & 45.2 & & 74 & 58.1 \\
\hline
\end{tabular}

Note $-N=$ frequencies $; A=$ accuracy.

Table 8

Accuracy (in Percent) and Frequencies as a Function of Eye Landing Time in the Window for Each Subject in Experiment 3

\begin{tabular}{cccccc}
\hline \multirow{2}{*}{$\begin{array}{c}\text { Landing } \\
\text { Time* }\end{array}$} & Subject 1 & & \multicolumn{2}{c}{ Subject 2 } \\
\cline { 2 - 3 } \cline { 5 - 6 } & N & A & & N & A \\
\hline $1-10$ & 27 & 44.4 & 15 & 46.7 \\
$11-20$ & 32 & 37.5 & 13 & 46.2 \\
$21-30$ & 14 & 50.0 & 20 & 45.0 \\
$31-40$ & 15 & 20.0 & 8 & 50.0 \\
$41-50$ & 12 & 58.3 & 10 & 50.0 \\
\hline
\end{tabular}

Note $-N=$ frequencies $; A=$ accuracy. $\quad$ *In milliseconds. 
Table 9

Percentage of Error Responses From Each Frame of the Matrix for Window and Control Trials in

Experiment 3

\begin{tabular}{lll}
\hline & \multicolumn{3}{c}{ Subject } \\
\cline { 2 - 3 } & 1 & 2 \\
\hline
\end{tabular}

Window Trials

$\begin{array}{ccc}\text { Frame 1 } & 93.2 & 88.6 \\ \text { Frame 2 } & 6.8 & 11.4 \\ & \text { Control Trials } & \\ \text { Frame 1 } & 80.0 & 67.7 \\ \text { Frame 2 } & 20.0 & 32.3\end{array}$

indicating that information from frame 1 was lost once a saccade was executed.

In sum, making the integration task even easier and increasing vertical and horizontal interdot distances still failed to produce integration. Thus, as in the two previous experiments, no evidence was found for the existence of an integrative visual buffer.

\section{GENERAL DISCUSSION}

The results of Experiments 1 and 2 suggest that the successful integration found by Jonides et al. (1982) was spurious, an artifact caused by phosphor persistence rather than by information stored in memory. In Experiment 3, an easier version of the integration task was used in another effort to demonstrate integration. While the overall level of accuracy was higher in this experiment, performance in the integration condition was still no better than it was in the control condition. Rayner and Pollatsek (1983) have also recently found that when phosphor persistence is eliminated as a contributing factor, little evidence for visual integration across saccades can be found.

Other reports in the literature suggesting the presence of integration may be questionable as well. For instance, the evidence for integration offered by Wolf et al. $(1978,1980)$ may have been due to persistence of their P-31 phosphor after its nominal offset. Although the P-31 (like the P-4 employed by Jonides et al., 1982) decays to $1 \%$ of its maximum brightness in less than $500 \mu \mathrm{sec}$, there is good reason to believe that visual information remains on the screen for several 10 s of milliseconds after offset. This is because the decay of the P-31, like that of the $\mathrm{P}-4$, is substantially slower after this initial $500-\mu \mathrm{sec}$ period, and the image must fall far below $1 \%$ of its maximum brightness to be below threshold under standard viewing conditions. This reduces our confidence in these and other results employing similar phosphors when immediate stimulus offset is required.

The results of the experiments reported in this paper cast doubt on the existence of an integrative visual buffer, a hypothetical storage site capable of underlying the spatial reconciliation and integration of visual information from successive fixations. However, it is possible that the task used in the present set of experiments was simply inappropriate to demonstrate integration. There are three obvious factors that may have been at fault. One is that the visual characteristics of the display and the timing parameters that were used may not have been appropriate for integration to occur. The second is the meaningless nature of the stimuli that were used (i.e., dot patterns); given the richness of ordinary visual environments, perhaps meaning is vital to integration. Finally, the fact that the two halves of the target matrix were presented in two separate frames of time, with the display shifting from one to the other during the saccade, may have inhibited integration. After all, the world rarely changes during a saccade. Thus, this feature of the task seems particularly troublesome.

But this is not to say that the results reported here are without value; on the contrary, they play an important role in restricting the space of possible integration mechanisms that one might propose. It is now clear that the visual contents of one fixation are not arbitrarily and automatically preserved across an eye movement and then combined with the contents of a new visual display. Thus, the integration mechanism, if there is one, does not passively construct a jigsaw mosaic of the visual world from independent visual "snapshots" of separate fixations. How integration does occur, assuming that it does, must depend on meaningful features of the visual environment and/or environmental constancy during a saccade. Further research focusing on these facets of the integration problem may yet bear fruitful results.

It is possible, however, that pursuit of the integrative visual buffer, at least as it has been described here, is misguided. Two arguments support this conclusion. One is that visual information from successive fixations differs widely in quality. Since acuity, for example, is much poorer in the periphery than in the fovea, any integrative visual buffer would have to combine pieces of visual information that differ qualitatively (e.g., a "fuzzy" chair from the periphery of one fixation with a clear image of the same chair now at the fovea). Related to this point, the retinal sizes and shapes of objects in the periphery and at the fovea are different; thus, any integration mechanism would have to deal with these discrepancies before combining information from successive fixations. One way in which this might be accomplished is by the integration of only low spatial frequency information from the periphery and the fovea. But this mechanism is very different from a "snapshot"--construction view of the integration process.

The second reason for questioning the viability of the integrative visual buffer construct is that it is not really necessary to explain our perception of the vi- 
sual world. The question raised at the beginning of this paper was how the world appeared stable and continuous despite temporally discontinuous visual input. All that is really required of the human perceptual system is the capability to ignore retinal image shifts that accompany saccades, and to override the retinal coordinates of previous fixations. During fixations, the image on the retina is (relatively) stable and continuous. Since saccadic suppression effectively eliminates visual input during a saccade, and previous fixations are masked by fixations (Davidson et al., 1973), there may be no need to postulate an integrative visual buffer to explain visual stability. This is not to deny that some spatial information is preserved across saccades; it appears, however, that visual images of successive fixations are not spatially aligned and fused in memory.

\section{REFERENCES}

Breitmeyer, B. G., Kropfl, W., \& Julesz, B. The existence and role of retinotopic and spatiotopic forms of visual persistence. Acta Psychologica, 1982, 52, 175-196.

Campbell, F. W., \& Wurtz, R. H. Saccadic omission: Why we do not see a gray-out during a saccadic eye movement. Vision Research, 1978, 18, 1297-1303.

Davidson, M. I., Fox, M. J., \& Dick, A. O. Effect of eye movements on backward masking and perceived location. Perception \& Psychophysics, 1973, 14, 110-116.

Dr Lollo, V. Temporal characteristics of iconic memory. Nature, $1977,267,241-243$.

DI LoLLo, V. Temporal integration in visual memory. Journal of Experimental Psychology: General, 1980, 109, 75-97.

Helmholtz, H. von. Handbuch der physiologischen Optik (Vol. 3). Leipzig, Germany: Voss, 1866.
Jonides, J., 'RWIN, D. E., \& Yantis, S. Integrating visual information from successive fixations. Science, 1982, 215, 192-194.

Jonides, J., Inwin, D. E., \& Yantis, S. Failure to integrate information from successive fixations. Science, in press.

McConkie, G. W., \& Rayner, K. Identifying the span of the effective stimulus in reading: Literature review and theories of reading. In H. Singer \& R. B. Ruddell (Eds.), Theoretical models and processes of reading. Newark, Del: International Reading Association, 1976.

McConkie, G. W., \& ZolA, D. Is visual information integrated across successive fixations in reading? Perception \& Psychophysics, 1979, 25, 221-224.

NeIsser, U. Cognitive psychology. Englewood Cliffs, N.J: Prentice-Hall, 1967.

RAYner, K., McConkie, G. W., \& Zola, D. Integrating information across eye movements. Cognitive Psychology, 1980, 12, 206-226.

Rayner, K., \& Pollatsek, A. Is visual information integrated across saccades? Perception \& Psychophysics, 1983, 34, 39-48.

RitTe R, M. Evidence for visual persistence during saccadic eye movements. Psychological Research, 1976, 39, 67-85.

Robinson, D. A. The mechanics of human saccadic eye movement. Journal of Physiology, 1964, 174, 245-264.

Wolf, W., Hauske, G., \& LuPp, U. How pre-saccadic gratings modify post-saccadic modulation transfer functions. Vision Research, 1978, 18, 1173-1179.

WolF, W., HAUSke, G., \& LuPP, U. Interactions of pre- and post-saccadic patterns having the same coordinates in space. Vision Research, 1980, 20, 117-125.

\section{NOTE}

1. By comparison, the illuminance of the display used by Jonides et al. (1982) was $4.52 \mathrm{~lx}$, with the same background luminance.

(Manuscript received January 27, 1983; revision accepted for publication April 6, 1983.) 